Volume 28 (2020) 127-140

DOI: $10.24330 /$ ieja.768202

\title{
ENDOMORPHISMS WITH CENTRAL VALUES ON PRIME RINGS WITH INVOLUTION
}

\author{
L. Oukhtite, H. EL Mir and B. Nejjar \\ Received: 1 October 2019; Revised: 6 December 2019; Accepted: 29 December 2019 \\ Communicated by A. Çiğdem Özcan
}

\begin{abstract}
In this paper we present some commutativity theorems for prime rings $R$ with involution $*$ of the second kind in which endomorphisms satisfy certain algebraic identities. Furthermore, we provide examples to show that various restrictions imposed in the hypotheses of our theorems are not superfluous.
\end{abstract}

Mathematics Subject Classification (2020): 16N60, 16W10, 16W25

Keywords: Prime ring, involution, commutativity, endomorphism

\section{Introduction}

Throughout this article, $R$ will represent an associative ring with center $Z(R)$. For any $x, y \in R$, the symbol $[x, y]$ will denote the commutator $x y-y x$; while the symbol $x \circ y$ will stand for the anti-commutator $x y+y x . R$ is 2 -torsion free if whenever $2 x=0$, with $x \in R$ implies $x=0 . R$ is prime if $a R b=0$ implies $a=0$ or $b=0$. An additive mapping $*: R \longrightarrow R$ is called an involution if $*$ is an anti-automorphism of order 2 ; that is $\left(x^{*}\right)^{*}=x$ and $(x y)^{*}=y^{*} x^{*}$ for all $x, y \in R$. An element $x$ in a ring with involution $(R, *)$ is said to be hermitian if $x^{*}=x$ and skew-hermitian if $x^{*}=-x$. The sets of all hermitian and skewhermitian elements of $R$ will be denote by $H(R)$ and $S(R)$, respectively. The involution is said to be of the first kind if $Z(R) \subseteq H(R)$, otherwise it is said to be of the second kind. In the latter case $S(R) \cap Z(R) \neq\{0\}$. A derivation on $R$ is an additive mapping $d: R \longrightarrow R$ such that $d(x y)=d(x) y+x d(y)$ for all $x, y \in R$. In [10], Brešar introduced the notion of generalized derivations in rings: an additive mapping $F: R \longrightarrow R$ is called generalized derivation if there exists a derivation $d$ such that $F(x y)=F(x) y+x d(y)$ holds for all $x, y \in R$, and $d$ is called the associated derivation of $F$. Over the last 30 years, several authors have investigated the relationship between the commutativity of the ring $R$ and certain special types of mappings on $R$. The first result in this direction is due to Divinsky [14], who proved that the simple artinian ring must be commutative if it has a commuting 
nontrivial automorphism. Two years later, Posner [21] proved that the existence of nonzero centralizing derivation on prime ring forces the ring to be commutative. Over the last few decades, several authors have subsequently refined and extended these result in various directions ([3], [4], [8], [19], [20] where further references can be found).

We say that a map $f: R \longrightarrow R$ preserves commutativity if $[f(x), f(y)]=$ 0 whenever $[x, y]=0$ for all $x, y \in R$. The study of commutativity preserving mappings has been an active research area in matrix theory, operator theory and ring theory (see [9], [22] for references). A map $f: R \longrightarrow R$ is said to be strong commutativity preserving $(S C P)$ on a subset $S$ of $R$ if $[f(x), f(y)]=[x, y]$ for all $x, y \in S$. In [6], Bell and Daif investigated the commutativity in rings admitting a derivation which is $S C P$ on a nonzero right ideal. Indeed, they proved that if a semiprime ring $R$ admits a derivation $d$ satisfying $[d(x), d(y)]=[x, y]$ for all $x, y$ in a right ideal $I$ of $R$, then $I \subseteq Z(R)$. In particular, $R$ is commutative if $I=R$. Later, Deng and Ashraf [13] proved that if there exist a derivation $d$ of a semiprime ring $R$ and a map $f: I \longrightarrow R$ defined on a nonzero ideal $I$ of $R$ such that $[f(x), d(y)]=[x, y]$ for all $x, y \in I$, then $R$ contains a nonzero central ideal. In particular, they showed that $R$ is commutative if $I=R$. Further, Ali and Huang [1] showed that if $R$ is a 2-torsion free semiprime ring an $d$ is a derivation of $R$ satisfying $[d(x), d(y)]+[x, y]=0$ for all $x, y$ in a nonzero ideal $I$ of $R$, then $R$ contains a nonzero central ideal. Many related generalizations of these results can be found in the literature (see for instance [2], [5], [7], [11], [12], [15], [17]).

Our aim in the present paper is to continue this line of investigation that consists on the study some special endomorphisms and their connection with commutativity for prime rings.

\section{Commutativity and $P_{i}$-endomorphisms}

In [16] it is proved that the prime ring $R$ must be commutative if $R$ is equipped with a generalized derivation $F$ associated with a nonzero derivation $d$ satisfying any one of the following conditions: (i) $F(x y)-[x, y] \in Z(R)$, (ii) $F(x y)-x \circ y \in Z(R)$, (iii) $F(x) F(y)-[x, y] \in Z(R)$, (iv) $F(x y)-x \circ y \in Z(R)$ for all $x, y \in I$, where $I$ is a nonzero two sided ideal of $R$.

Motivated by the previous results, our purpose in this section is to examine what happens in case the generalized derivation $F$ is replaced by an endomorphism $g$ satisfying any one of the following properties:

$$
g(x y)-[x, y] \in Z(R) \text { for all } x, y \in R \quad\left(P_{1}\right)
$$




$$
\begin{array}{ll}
g(x y)+[x, y] \in Z(R) \text { for all } x, y \in R & \left(P_{2}\right) \\
g(x y)-x \circ y \in Z(R) \text { for all } x, y \in R & \left(P_{3}\right) \\
g(x y)+x \circ y \in Z(R) \text { for all } x, y \in R . & \left(P_{4}\right)
\end{array}
$$

More precisely, we will discuss existence of such mappings and their relationship with commutativity in case of prime rings.

Definition 2.1. Let $R$ be a ring and $g: R \longrightarrow R$ an endomorphism. For $i \in$ $\{1,2,3,4\}$ we will say that $g$ is a $P_{i}$-endomorphism if $g$ satisfies the property $P_{i}$.

Remark 2.2. If $R$ is a commutative ring, then every endomorphism is a $P_{i^{-}}$ endomorphism for $i \in\{1,2,3,4\}$.

Our next aim is to give examples of $P_{i}$-endomorphisms over noncommutative rings.

Example 2.3. (1) Let $\mathcal{A}=\left\{\left(\begin{array}{ll}a & b \\ 0 & c\end{array}\right) \mid a, b, c \in \mathbb{Z}\right\}$ and $\mathcal{B}=\left\{\left(\begin{array}{ll}0 & t \\ 0 & 0\end{array}\right) \mid t \in \mathbb{Z}\right\}$. If we take $\mathcal{R}=\left\{\left(\begin{array}{cc}0 & 0 \\ A & B\end{array}\right) \mid A \in \mathcal{A}, B \in \mathcal{B}\right\}$ then for $X, Y \in \mathcal{R}$ such that

$$
\begin{gathered}
X=\left(\begin{array}{ll}
0 & 0 \\
A & B
\end{array}\right) \text { and } Y=\left(\begin{array}{cc}
0 & 0 \\
A^{\prime} & B^{\prime}
\end{array}\right) \text {, we have } \\
X Y=\left(\begin{array}{cc}
0 & 0 \\
B A^{\prime} & B B^{\prime}
\end{array}\right)=\left(\begin{array}{cc}
0 & 0 \\
B A^{\prime} & 0
\end{array}\right) \in Z(\mathcal{R})
\end{gathered}
$$

then it is obvious to verify that the endomorphism $g$ defined on the noncommutative ring $\mathcal{R}$ by:

$$
g\left(\begin{array}{cc}
0 & 0 \\
A & B
\end{array}\right)=\left(\begin{array}{cc}
0 & 0 \\
\alpha A & B
\end{array}\right),
$$

where $\alpha \in \mathbb{Z}$, is a $P_{i}$-endomorphism for $i \in\{1,2,3,4\}$.

(2) Let $\mathcal{S}=\mathcal{R} \times \mathcal{C}$ where $\mathcal{C}$ is a commutative ring and $\mathcal{R}$ is the ring provided with the endomorphism $g$ defined in the example (1). It is straightforward to check that $G: S \longrightarrow S$ defined by:

$$
G(R, C)=(g(R), h(C)),
$$

where $h$ is any endomorphism defined on $\mathcal{C}$, is a $P_{i}$-endomorphism for $i \in\{1,2,3,4\}$.

The following theorem gives some commutativity criteria through the behavior of the $P_{i}$-endomorphisms.

Theorem 2.4. Let $R$ be a prime ring and $g$ an endomorphism of $R$. The following assertions are equivalent: 
(1) $g$ is a $P_{1}$-endomorphism;

(2) $g$ is a $P_{2}$-endomorphism;

(3) $g$ is a $P_{3}$-endomorphism;

(4) $g$ is a $P_{4}$-endomorphism;

(5) $R$ is a commutative integral domain.

Proof. For the non-trivial implications assume that $R$ is not commutative. $(1) \Rightarrow(5)$ We are given that

$$
g(x) g(y)-[x, y] \in Z(R) \text { for all } x, y \in R .
$$

If $Z(R)=\{0\}$, then (1) reduces to

$$
g(x) g(y)-[x, y]=0 \text { for all } x, y \in R .
$$

Substituting $y x$ for $y$ in equation (2) one can see that

$$
[x, y](g(x)-x)=0 \text { for all } x, y \in R,
$$

in such a way that $[x, y] R(g(x)-x)=0$ for all $x, y \in R$.

Using the primeness of $R$ together with Brauer's trick (that is a group cannot be a union of two of its proper subgroups), we conclude that $g=I_{d}$. Hence (2) yields $y x=0$ and therefore $R=\{0\}$. Consequently $Z(R) \neq\{0\}$; replacing $y$ by $y z^{2}$ in (1), where $z \in Z(R) \backslash\{0\}$, we arrive at $[[x, y], r]\left(g\left(z^{2}\right)-z^{2}\right)=0$ and therefore

$$
[[x, y], r] R\left(g\left(z^{2}\right)-z^{2}\right)=0 \text { for all } r, x, y \in R .
$$

Since $R$ is prime, then either $[[x, y], r]=0$ or $g\left(z^{2}\right)-z^{2}=0$.

Assume that $[[x, y], r]=0$ for all $r, x, y \in R$; replacing $y$ by $y x$ in the last expression, we obtain $[x, y][x, r]=0$ which leads to $[x, y] R[x, y]=0$ for all $x, y \in R$. Once again invoking the primeness of $R$ we get $[x, y]=0$ for all $x, y \in R$ which contradicts the fact that $R$ is not commutative. Hence $g\left(z^{2}\right)=z^{2}$, and thus taking $y=z^{2}$ in the hypothesis, we obtain

$$
g(x) z^{2} \in Z(R) \text { for all } x \in R
$$

which implies that $z^{2}=0$ or $g(x) \in Z(R)$. Since $z \neq 0$, then $g(x) \in Z(R)$ and our hypothesis reduces to $[x, y] \in Z(R)$ for all $x, y \in R$, so that

$$
[[x, y], r]=0 \text { for all } r, x, y \in R,
$$

which, as above, contradicts the fact that $R$ is not commutative. Then, we conclude that $R$ is a commutative integral domain.

$(2) \Rightarrow(5)$ Follows from the first implication with a slight modification. 
$(3) \Rightarrow(5)$ Suppose that

$$
g(x) g(y)-x \circ y \in Z(R) \text { for all } x, y \in R .
$$

If $Z(R)=\{0\}$, then our assumption becomes

$$
g(x) g(y)-x \circ y=0 \text { for all } x, y \in R .
$$

Substituting $y x$ for $y$ in equation (6), we arrive at

$$
(x \circ y)(g(x)-x)=0 \text { for all } x, y \in R
$$

replacing $y$ by $y r$, we obviously get

$$
(y(x \circ r)+[x, y] r)(g(x)-x)=0 \text { for all } r, x, y \in R .
$$

By view of equation (7), equation (8) yields

$$
[x, y] R(g(x)-x)=0 \text { for all } x, y \in R .
$$

Since $R$ is not commutative then $g=I_{d}$ and the hypothesis becomes

$$
y x=0 \text { for all } x, y \in R,
$$

and thus $R=\{0\}$, a contradiction. Hence $Z(R) \neq\{0\}$; replacing $y$ by $y z^{2}$ in equation (5), where $z \in Z(R) \backslash\{0\}$, we arrive at

$$
[x \circ y, r]\left(g\left(z^{2}\right)-z^{2}\right)=0 \text { for all } r, x, y \in R .
$$

Since $R$ is not commutative, then $g\left(z^{2}\right)=z^{2}$; replacing $y$ by $z^{2}$ in the hypothesis, we find that

$$
(g(x)-2 x) z^{2} \in Z(R) \text { for all } x \in R
$$

which implies that $z^{2}=0$ or $g(x)-2 x \in Z(R)$. Accordingly $g(x)-2 x \in Z(R)$; substituting $x y$ for $x$ we obtain

$$
g(x) g(y)-2 x y \in Z(R) \text { for all } x, y \in R .
$$

Using the last equation together with equation (5) one can see that

$$
[x, y] \in Z(R) \text { for all } x, y \in R
$$

hence $R$ is commutative, a contradiction. We conclude that $R$ is a commutative integral domain.

$(4) \Rightarrow(5)$ Follows from the last implication with a slight modification.

The following example proves that the primeness hypothesis of $R$ is necessary in Theorem 2.4. 
Example 2.5. Let $\mathcal{R}=\left\{\left(\begin{array}{ll}0 & 0 \\ A & B\end{array}\right) \mid A \in \mathcal{A}, B \in \mathcal{B}\right\}$, where

$$
\mathcal{A}=\left\{\left(\begin{array}{ll}
a & b \\
0 & c
\end{array}\right) \mid a, b, c \in \mathbb{Z}\right\} \text { and } \mathcal{B}=\left\{\left(\begin{array}{ll}
0 & t \\
0 & 0
\end{array}\right) \mid t \in \mathbb{Z}\right\} .
$$

$\mathcal{R}$ is not prime, because of $X Y Z=0$ for all $X, Y, Z \in \mathcal{R}$. Moreover, for all $X, Y \in \mathcal{R}$ we have $[X, Y] \in Z(\mathcal{R})$ and $X \circ Y \in Z(\mathcal{R})$. Hence the zero endomorphism $\Theta_{\mathcal{R}}$ is a $P_{i}$-endomorphism for $i \in\{1,2,3,4\}$ but $R$ is not commutative.

\section{Commutativity and $P_{i}^{*}$-endomorphisms}

The authors in [16] explore the commutativity of a prime ring with involution $(R, *)$ provided with a generalized derivation $F$ satisfying any one of the following conditions:

(i) $F\left(x x^{*}\right)-\left[x, x^{*}\right] \in Z(R), \quad$ (ii) $F\left(x x^{*}\right)-x \circ x^{*} \in Z(R)$,

(iii) $F(x) F\left(x^{*}\right)-\left[x, x^{*}\right] \in Z(R), \quad$ (iv) $F(x) F\left(x^{*}\right)-x \circ x^{*} \in Z(R)$

for all $x \in R$. Our purpose in this section is to continue this line of investigation by studying commutativity criteria for rings with involution admitting an endomorphism satisfying certain algebraic identities in a more general situation. More precisely, we will establish an endomorphism version of the above properties.

Therefore, we need to introduce more general classes of mappings as in the following definition.

Definition 3.1. Let $(R, *)$ be a ring with involution and let $g: R \longrightarrow R$ be an endomorphism. Then $g$ is called a:

(1) $P_{1}^{*}$-endomorphism if $g\left(x x^{*}\right)-\left[x, x^{*}\right] \in Z(R)$ for all $x \in R$.

(2) $P_{2}^{*}$-endomorphism if $g\left(x x^{*}\right)+\left[x, x^{*}\right] \in Z(R)$ for all $x \in R$.

(3) $P_{3}^{*}$-endomorphism if $g\left(x x^{*}\right)-x \circ x^{*} \in Z(R)$ for all $x \in R$.

(4) $P_{4}^{*}$-endomorphism if $g\left(x x^{*}\right)+x \circ x^{*} \in Z(R)$ for all $x \in R$.

Example 3.2. (1) Let us consider $R=M_{2}(\mathbb{R})$ and define an involution on $R$ by setting

$$
\left(\begin{array}{ll}
a & b \\
c & d
\end{array}\right)^{*}=\left(\begin{array}{cc}
d & -b \\
-c & a
\end{array}\right)
$$

Since $\left[X, X^{*}\right]=0$ and $X \circ X^{*} \in Z(R)$ for all $X \in R$, then the zero function $\Theta_{R}$ is a $P_{i}^{*}$-endomorphism for $i \in\{1,2,3,4\}$.

Also, if we take $g: R \longrightarrow R$ the endomorphism defined by $g(X)=P X P^{-1}$ with $P=\left(\begin{array}{ll}3 & 0 \\ 0 & 1\end{array}\right)$, then for all $X \in R$ we have $g\left(X X^{*}\right)=X X^{*}=X^{*} X \in Z(R)$ so 
that $g$ is a $P_{i}^{*}$-endomorphism for $i \in\{1,2,3,4\}$.

(2) Let us consider $R=\left\{\left(\begin{array}{ll}a & b \\ 0 & c\end{array}\right) \mid a, b, c \in \mathbb{R}\right\}$ provided with the involution of the first kind $\left(\begin{array}{ll}a & b \\ 0 & c\end{array}\right)^{*}=\left(\begin{array}{cc}c & -b \\ 0 & a\end{array}\right)$ and let $g: R \longrightarrow R$ be the endomorphism defined by: $g\left(\begin{array}{ll}a & b \\ 0 & c\end{array}\right)=\left(\begin{array}{cc}a & a-c+2 b \\ 0 & c\end{array}\right)$.

Set $X=\left(\begin{array}{cc}a & b \\ 0 & c\end{array}\right)$, we have $X X^{*}=X^{*} X=\left(\begin{array}{cc}a c & 0 \\ 0 & a c\end{array}\right)$ so that $\left[X, X^{*}\right]=0$ and $X \circ X^{*} \in Z(R)$. Hence $g\left(X X^{*}\right)=X X^{*} \in Z(R)$ and thus $g$ is a $P_{i}^{*}$-endomorphism for $i \in\{1,2,3,4\}$.

(3) Let $(R, *)$ be as in the example (2) and consider $\mathcal{R}=R \times \mathbb{C}$ provided with the involution of the second kind defined by $\sigma(X, Z)=\left(X^{*}, \bar{Z}\right)$.

Set $\mathcal{X}=(X, Z) \in \mathcal{R}$, one can easily verify that $[\mathcal{X}, \sigma(\mathcal{X})]=\left(\left[X, X^{*}\right],[Z, \bar{Z}]\right)=0_{\mathcal{R}}$ and $\mathcal{X} \circ \sigma(\mathcal{X})=\left(X \circ X^{*}, Z \circ \bar{Z}\right) \in Z(R)$ hence the zero endomorphism $\Theta_{\mathcal{R}}$ is a $P_{i}^{*}$-endomorphism for $i \in\{1,2,3,4\}$.

The questions that now arise are:

(1) Can we characterize the commutativity of a ring with involution $(R, *)$ by using the properties $P_{i}^{*}$ for all $i \in\{1,2,3,4\}$ ?

(2) Is there a relation between $P_{i}^{*}$ and $P_{i}$ for all $i \in\{1,2,3,4\}$ ?

The next examples show that $P_{i}^{*}$ are not $P_{i}$ in each of the following situations.

Example 3.3 (Counter-examples). (1) Let $(R, *)$ be as in Example $3.2(1)$. It is straightforward to check that $R$ is a prime ring and $*$ is an involution of the first kind. Moreover, for $X=\left(\begin{array}{ll}1 & 1 \\ 0 & 0\end{array}\right)$ and $Y=\left(\begin{array}{ll}1 & 1 \\ 0 & 1\end{array}\right)$, we have $[X, Y]=\left(\begin{array}{ll}0 & 1 \\ 0 & 0\end{array}\right) \notin Z(R)$ and $X \circ Y=\left(\begin{array}{ll}2 & 3 \\ 0 & 0\end{array}\right) \notin Z(R)$, then the zero function $\Theta_{R}$ is not a $P_{i}$-endomorphism for $i \in\{1,2,3,4\}$.

(2) Let consider $(R, *)$ provided with the endomorphism defined in Example 3.2

(2). One can easily verify that $R$ is not prime and $*$ is an involution of the first kind. Moreover, for $X=\left(\begin{array}{ll}1 & 1 \\ 0 & 0\end{array}\right)$ and $Y=\left(\begin{array}{ll}1 & 1 \\ 0 & 1\end{array}\right)$, we find that $g(X Y)=\left(\begin{array}{ll}1 & 3 \\ 0 & 0\end{array}\right)$. Hence it is clear that $g$ is not a $P_{i}$-endomorphism for $i \in\{1,2,3,4\}$.

(3) Let consider $(\mathcal{R}, \sigma)$ the ring defined in Example $3.2(3)$. $\mathcal{R}$ is not prime 
and $\sigma$ is an involution of the second kind. Set $\mathcal{X}=\left(\left(\begin{array}{ll}1 & 1 \\ 0 & 0\end{array}\right), 1\right)$ and $\mathcal{Y}=$ $\left(\left(\begin{array}{ll}1 & 1 \\ 0 & 1\end{array}\right), 1\right) \in \mathcal{R}$, then we have $[\mathcal{X}, \mathcal{Y}]=\left(\left(\begin{array}{ll}0 & 1 \\ 0 & 0\end{array}\right), 0\right) \notin Z(\mathcal{R})$ and $\mathcal{X} \circ \mathcal{Y}=$ $\left(\left(\begin{array}{ll}2 & 3 \\ 0 & 0\end{array}\right), 2\right) \notin Z(\mathcal{R})$. Hence the zero function $\Theta_{\mathcal{R}}$ is not a $P_{i}$-endomorphism for $i \in\{1,2,3,4\}$.

We are not able to find an example of a $P_{i}^{*}$-endomorphism which is not a $P_{i^{-}}$ endomorphism in the case where $(R, *)$ is a prime ring with involution of the second kind. Consequence of which it's natural to consider the following conjecture:

Conjecture: Let $(R, *)$ be a 2 -torsion free prime ring with involution of the second kind and $g$ an endomorphism of $R$. Then $g$ is a $P_{i}^{*}$-endomorphism if and only if $g$ is a $P_{i}$-endomorphism for $i \in\{1,2,3,4\}$.

The next theorem gives an affirmative answer to the above conjecture. However, we will use frequently the following Lemma which is very crucial for developing the proofs of our theorem.

Lemma 3.4. Let $(R, *)$ be a 2-torsion free semiprime ring with involution of the second kind and $a \in R$.

(i) If $\left[\left[x, x^{*}\right], a\right]=0$ for all $x \in R$, then $a \in Z(R)$.

(ii) If $\left[x \circ x^{*}, a\right]=0$ for all $x \in R$, then $a \in Z(R)$.

Proof. (i) Let $a \in R$, we are given that

$$
\left[\left[x, x^{*}\right], a\right]=0 \text { for all } x \in R .
$$

Linearizing this equation and replacing $y$ by $y^{*}$, we find that

$$
[[x, y], a]+\left[\left[y^{*}, x^{*}\right], a\right]=0 \text { for all } x, y \in R .
$$

Substituting $y s$ for $y$, where $s \in S(R) \cap Z(R)$, and using the last equation, we obtain

$$
[[x, y], a]=0 \text { for all } x, y \in R .
$$

Replacing $y$ by $y x$ one can see that

$$
[x, y][x, a]=0 \text { for all } x, y \in R .
$$

Writing ay instead of $y$, we arrive at

$$
[x, a] y[x, a]=0 \text { for all } x, y \in R
$$


and thus

$$
[x, a] R[x, a]=0 \text { for all } x \in R .
$$

Since $R$ is semiprime we deduce that $[x, a]=0$ for all $x \in R$, hence $a \in Z(R)$.

(ii) Let $a \in R$, we are given that

$$
\left[x \circ x^{*}, a\right]=0 \text { for all } x \in R .
$$

Linearizing this equation and replacing $y$ by $y^{*}$, we find that

$$
[x \circ y, a]+\left[y^{*} \circ x^{*}, a\right]=0 \text { for all } x, y \in R .
$$

Substituting $y s$ for $y$ and using (18), we obtain

$$
[x \circ y, a]=0 \text { for all } x, y \in R .
$$

Taking $y=z$ where $z \in Z(R) \backslash\{0\}$ we arrive at

$$
[x, a]=0 \text { for all } x \in R .
$$

Then we conclude that $a \in Z(R)$.

Theorem 3.5. Let $(R, *)$ be a 2-torsion free prime ring with involution of the second kind. If $g$ is a non-trivial endomorphism of $R$, then $g$ is a $P_{i}^{*}$-endomorphism if and only if $g$ is a $P_{i}$-endomorphism for $i \in\{1,2,3,4\}$.

Proof. For the non-trivial implications, we need to prove that $g$ is a $P_{i}^{*}$-endomorphism implies that $g$ is a $P_{i}$-endomorphism for $i \in\{1,2,3,4\}$.

1) Assume that $g$ is a $P_{1}^{*}$-endomorphism, then

$$
g(x) g\left(x^{*}\right)-\left[x, x^{*}\right] \in Z(R) \text { for all } x \in R .
$$

Linearizing equation (21) and replacing $y$ by $y^{*}$, we find that

$$
g(x) g(y)+g\left(y^{*}\right) g\left(x^{*}\right)-[x, y]-\left[y^{*}, x^{*}\right] \in Z(R) \text { for all } x, y \in R .
$$

Replacing $y$ by $y h$ in (22), where $h \in Z(R) \cap H(R)$, we obtain

$$
\left(g(x) g(y)+g\left(y^{*}\right) g\left(x^{*}\right)\right) g(h)-\left([x, y]+\left[y^{*}, x^{*}\right]\right) h \in Z(R) \text { for all } x, y \in R .
$$

Using equation (21), where $h \in Z(R) \cap H(R)$, one can see that

$$
\left[\left[x, x^{*}\right], g(h)\right]=0 \text { for all } x \in R .
$$

Then by Lemma 3.4, equation (24) yields

$$
g(h) \in Z(R) \text { for all } h \in Z(R) \cap H(R),
$$

and therefore (22) becomes

$$
\left(g(x) g(y)+g\left(y^{*}\right) g\left(x^{*}\right)\right) g(h)-\left([x, y]+\left[y^{*}, x^{*}\right]\right) g(h) \in Z(R) \text { for all } x, y \in R .
$$


Combining (23) together with (25) it follows that

$$
\left([x, y]+\left[y^{*}, x^{*}\right]\right)(g(h)-h) \in Z(R) \text { for all } x, y \in R .
$$

Substituting $x^{*}$ for $y$ we get

$$
\left[x, x^{*}\right](g(h)-h) \in Z(R) \text { for all } x \in R .
$$

Invoking the primeness of $R$, the last relation implies that either $\left[x, x^{*}\right] \in Z(R)$ or $g(h)-h=0$. By [18, Lemma 2.1], if $\left[x, x^{*}\right] \in Z(R)$, for all $x \in R$, we deduce that $R$ is commutative and therefore $g$ is a $P_{1}$-endomorphism. If $g(h)=h$ for all $h \in Z(R) \cap H(R)$ then $g(s)=s$ or $g(s)=-s$ for all $s \in Z(R) \cap S(R)$.

Assume that $g(s)=s$; replacing $y$ by $y s$ in equation (22) we find that

$$
g(x) g(y)-g\left(y^{*}\right) g\left(x^{*}\right)-[x, y]+\left[y^{*}, x^{*}\right] \in Z(R) \text { for all } x, y \in R .
$$

Using equation (26) together with equation (22), it follows that

$$
g(x) g(y)-[x, y] \in Z(R) \text { for all } x, y \in R
$$

and thus $g$ is a $P_{1}$-endomorphism.

If $g(s)=-s$ for all $s \in S(R) \cap Z(R)$; substituting $y s$ for $y$ in equation (22) we arrive at

$$
-g(x) g(y)+g\left(y^{*}\right) g\left(x^{*}\right)-[x, y]+\left[y^{*}, x^{*}\right] \in Z(R) \text { for all } x, y \in R .
$$

Using (27) together with (22) we get

$$
g(y) g(x)-\left[x^{*}, y^{*}\right] \in Z(R) \text { for all } x, y \in R .
$$

Substituting $s$ for $y$, where $s \in Z(R) \cap S(R)$, one can see that

$$
g(x) \in Z(R) \text { for all } x \in R
$$

thereby, the relation (21) gives $\left[x, x^{*}\right] \in Z(R)$ for all $x \in R$ and using [18, Lemma 2.1 ] we conclude that $R$ is commutative, then $g$ is a $P_{1}$-endomorphism.

2) Assume that $g$ is a $P_{2}^{*}$-endomorphism. Using similar argument as in the proof of 1 ) we can prove that $g$ is a $P_{2}$-endomorphism.

3) Suppose $g$ is a $P_{3}^{*}$-endomorphism, that is

$$
g(x) g\left(x^{*}\right)-x \circ x^{*} \in Z(R) \text { for all } x \in R .
$$

Then a linearization of (29) forces

$$
g(x) g(y)+g\left(y^{*}\right) g\left(x^{*}\right)-x \circ y-y^{*} \circ x^{*} \in Z(R) \text { for all } x, y \in R .
$$


Writing $y h$ instead of $y$ in the last equation, where $h \in Z(R) \cap H(R)$, we obtain

$$
\left(g(x) g(y)+g\left(y^{*}\right) g\left(x^{*}\right)\right) g(h)-\left(x \circ y+y^{*} \circ x^{*}\right) h \in Z(R) \text { for all } x, y \in R .
$$

By view of Lemma 3.4, equation (30) yields

$$
\left(g(x) g(y)+g\left(y^{*}\right) g\left(x^{*}\right)-x \circ y-y^{*} \circ x^{*}\right) g(h) \in Z(R) \text { for all } x, y \in R .
$$

Subtracting (31) from (32), we find that

$$
\left(x \circ y+y^{*} \circ x^{*}\right)(g(h)-h) \in Z(R) \text { for all } x, y \in R .
$$

Substituting $x^{*}$ for $y$ we get

$$
\left(x \circ x^{*}\right)(g(h)-h) \in Z(R) \text { for all } x \in R
$$

which implies that either $x \circ x^{*} \in Z(R)$ or $g(h)=h$ for all $h \in Z(R) \cap H(R)$.

If $x \circ x^{*} \in Z(R)$ for all $x \in R$ then $R$ is commutative by [18, Lemma 2.2], hence $g$ is a $P_{3}$-endomorphism. If $g(h)=h$ then $g(s)=s$ or $g(s)=-s$ for all $s \in Z(R) \cap S(R)$. Suppose that $g(s)=s$, then replacing $y$ by $y s$ in equation (30) we find that

$$
g(x) g(y)-g\left(y^{*}\right) g\left(x^{*}\right)-x \circ y+y^{*} \circ x^{*} \in Z(R) \text { for all } x, y \in R .
$$

Comparing equations (30) and (35), we are forced to conclude that

$$
g(x) g(y)-x \circ y \in Z(R) \text { for all } x, y \in R
$$

hence $g$ is a $P_{3}$-endomorphism.

If $g(s)=-s$ for all $s \in S(R) \cap Z(R)$; substituting $y s$ for $y$ in equation (30) we arrive at

$$
-g(x) g(y)+g\left(y^{*}\right) g\left(x^{*}\right)-x \circ y+y^{*} \circ x^{*} \in Z(R) \text { for all } x, y \in R .
$$

Invoking (30), the previous equation yields

$$
g(y) g(x)-x^{*} \circ y^{*} \in Z(R) \text { for all } x, y \in R .
$$

Putting $s$ instead of $y$, where $s \in Z(R) \cap S(R)$, one can see that

$$
g(x)-2 x^{*} \in Z(R) \text { for all } x \in R .
$$

Replacing $x$ by $y x$ in (38) and comparing with (37), we obviously get

$$
[x, y] \in Z(R) \text { for all } x, y \in R
$$

and thus

$$
\left[x, x^{*}\right] \in Z(R) \text { for all } x \in R .
$$

By [18, Lemma 2.1], we conclude that $R$ is commutative, so that $g$ is a $P_{3^{-}}$ endomorphism. 
4) Assume that $g$ is a $P_{4}^{*}$-endomorphism. Using similar argument as in the proof of 3 ) we can prove that $g$ is a $P_{4}$-endomorphism.

The following example proves that the condition "* is of the second kind" is necessary in Theorem 3.5.

Example 3.6. Let us consider $R=M_{2}(\mathbb{Z})$ and $\left(\begin{array}{ll}a & b \\ c & d\end{array}\right)^{*}=\left(\begin{array}{cc}d & -b \\ -c & a\end{array}\right)$. It is straightforward to check that $(R, *)$ is a prime ring and $*$ is an involution of the first kind. Moreover, for all $X \in R$, we have $\left[X, X^{*}\right]=0$ and $X \circ X^{*} \in Z(R)$.

Then the zero endomorphism $\Theta_{R}$ is a $P_{i}^{*}$-endomorphism for $i \in\{1,2,3,4\}$; but $\Theta_{R}$ is not a $P_{i}$-endomorphism for $i \in\{1,2,3,4\}$, since $R$ is non commutative.

In this second example we will show that Theorem 3.5 cannot be extended to semiprime rings.

Example 3.7. Let us consider $R=M_{2}(\mathbb{Z}) \times \mathbb{C}$ and $\sigma$ the involution of the second kind defined on $R$ by $\sigma(X, Z)=\left(X^{*}, \bar{Z}\right)$ with $*$ the involution defined in the latter example. The zero endomorphism $\Theta_{R}$ is a $P_{i}^{*}$-endomorphism for $i \in\{1,2,3,4\}$; but for

$$
X=\left(\left(\begin{array}{ll}
1 & 1 \\
0 & 0
\end{array}\right), 1\right), Y=\left(\left(\begin{array}{ll}
1 & 0 \\
1 & 0
\end{array}\right), 2\right) \text { and } Z=\left(\left(\begin{array}{ll}
0 & 1 \\
0 & 0
\end{array}\right), 3\right)
$$

one can verify that $[[X, Y], Z] \neq 0$ and $[X \circ Y, Z] \neq 0$. Hence $\Theta_{R}$ is not a $P_{i^{-}}$ endomorphism for $i \in\{1,2,3,4\}$.

Acknowledgement. The authors would like to thank the referee for the valuable suggestions and comments.

\section{References}

[1] S. Ali and S. Huang, On derivations in semiprime rings, Algebr. Represent. Theory, 15(6) (2012), 1023-1033.

[2] M. Ashraf and M. A. Quadri, Some conditions for the commutativity of rings, Acta Math. Hungar., 61(1-2) (1993), 73-77.

[3] M. Ashraf and N. Rehman, On commutativity of rings with derivations, Results Math., 42(1-2) (2002), 3-8.

[4] H. E. Bell, Some results on commutativity and anti-commutativity in rings, Acta Math. Hungar., 63(2) (1994), 113-117.

[5] H. E. Bell, A. Boua and L. Oukhtite, Semigroup ideals and commutativity in 3-prime near rings, Comm. Algebra, 43(5) (2015), 1757-1770. 
[6] H. E. Bell and M. N. Daif, On commutativity and strong commutativitypreserving maps, Canad. Math. Bull., 37(4) (1994), 443-447.

[7] H. E. Bell and M. N. Daif, On derivations and commutativity in prime rings, Acta Math. Hungar., 66(4) (1995), 337-343.

[8] H. E. Bell and W. S. Martindale, III, Centralizing mappings of semiprime rings, Canad. Math. Bull., 30(1) (1987), 92-101.

[9] M. Brešar, Commuting traces of biadditive mapping, commutativity preserving mapping and Lie mappings, Trans. Amer. Math. Soc., 335(2) (1993), 525-546.

[10] M. Brešar, On the distance of the composition of two derivations to the generalized derivation, Glasgow Math. J., 33(1) (1991), 89-93.

[11] M. Brešar and C. R. Miers, Strong commutativity preserving maps of semiprime rings, Canad. Math. Bull., 37(4) (1994), 457-460.

[12] V. De Filippis, A. Mamouni and L. Oukhtite, Generalized Jordan semiderivations in prime rings, Canad. Math. Bull., 58(2) (2015), 263-270.

[13] Q. Deng and M. Ashraf, On strong commutativity preserving mappings, Results Math., 30 (1996), 259-263.

[14] N. Divinsky, On commuting automorphisms of rings, Trans. Roy. Soc. Canada Sect. III, 49 (1955), 19-22.

[15] A. Fošner, X.-F. Liang and F. Wei, Centralizing traces with automorphisms on triangular algebras, Acta Math. Hungar., 154(2) (2018), 315-342.

[16] A. Mamouni, B. Nejjar and L. Oukhtite, Differential identities on prime rings with involution, J. Algebra Appl., 17(9) (2018), 1850163 (11 pp).

[17] A. Mamouni, L. Oukhtite and B. Nejjar, On *-semiderivations and *generalized semiderivations, J. Algebra Appl., 16(4) (2017), 1750075 (8 pp).

[18] B. Nejjar, A. Kacha, A. Mamouni and L. Oukhtite, Commutativity theorems in rings with involution, Comm. Algebra, 45(2) (2017), 698-708.

[19] L. Oukhtite, Posner's second theorem for Jordan ideals in rings with involution, Expo. Math., 29(4) (2011), 415-419.

[20] L. Oukhtite and A. Mamouni, Generalized derivations centralizing on Jordan ideals of rings with involution, Turkish J. Math., 38(2) (2014), 225-232.

[21] E. C. Posner, Derivations in prime rings, Proc. Amer. Math. Soc., 8 (1957), 1093-1100.

[22] P. Semrl, Commutativity preserving maps, Linear Algebra Appl., 429 (2008), 1051-1070. 
L. Oukhtite (Corresponding Author) and H. EL Mir Laboratory of Modeling and Mathematical Structures

Department of Mathematics

Faculty of Science and Technology

University S. M. Ben Abdellah

Fez, Morocco

e-mails: oukhtitel@hotmail.com (L. Oukhtite)

hajar.elmir@usmba.ac.ma (H. EL Mir)

\section{B. Nejjar}

Department of Mathematics

Faculty of Science

University Ibn Tofail

Kenitra, Morocco

e-mail: bader.nejjar@gmail.com 